
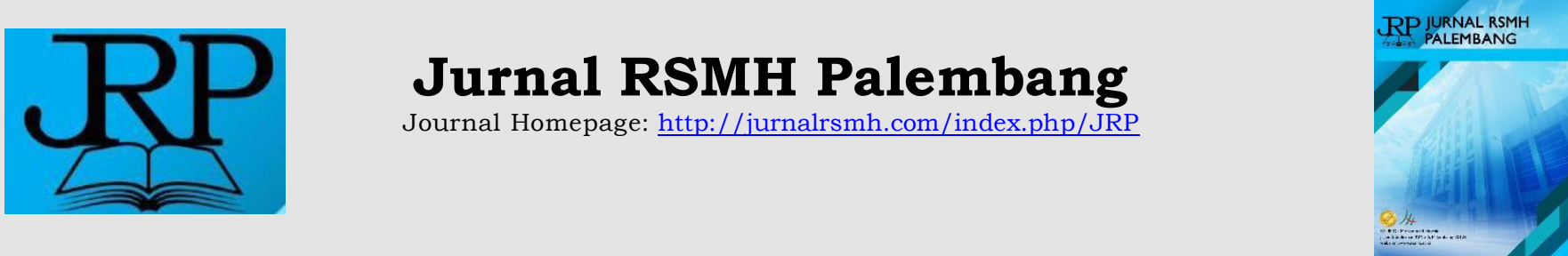

\title{
Evidence-based case report: Coinfection of COVID-19 in children and administration of antibiotics
}

\section{Fifi Sofiah, Marselya Ulfa, Azwar Aruf, Raden Muhammad Indra}

Pediatric Medical Staff Group dr. Mohammad Hoesin Palembang / Children's Section, Faculty of Medicine, Sriwijaya University

\author{
A R T I C L E I N F O \\ Keywords: \\ COVID-19 \\ Children \\ Coinfection \\ Antibiotic \\ Azithromycin.

\section{Corresponding author: Fifi Sofiah \\ E-mail address: fifisofiah@gmail.com}

All authors have reviewed and approved the final version of the manuscript.

https://doi.org/10.37275/JRP.v1i2.8

\begin{abstract}
A B S T R A C T
Background. COVID-19 in childrenalthoughmostly mild, but can also cause serious illness and even death. Coinfection, especially bacterialcan increase the severity of the disease. There is yet sufficient evidence about the role of antibiotics in childhood COVID-19 with coinfection.Objective. To review the available evidence on the coinfection in childhood COVID-19 and the role of antibiotic administration. Method.Online literature search using Pubmed database, google scholar dan Cohcrane library Results. No study was found that directly evaluate the efficacy of antibiotic in childhood COVID-19 with coinfection. Three meta-analyses found rates of coinfection of $5.6 \%-14 \%$ and one case series identified a very high rate $(94.2 \%)$. Most frequent pathogens included Mycoplasma pneumonia, Streptococcus pneumonia,and influenza/parainfluenza viruses. Two RCTsandone case series on antibiotic administration, but all three studies did not address coinfection status. All three studies evaluated the combination of azithromycin and hydroxychloroquine. No changes in illness severity or mortality attributed to the medications, one study indicated more rapid viral load clearance associated with azithromycin. Conclusion There is a lack of evidence on the role of antibiotics in the management of childhood COVID-19with coinfection. Azithromycin can be considered in some cases.
\end{abstract}

\section{Introduction}

Coronavirus disease 2019 (COVID-19) is a respiratory infection caused by the severe acute respiratory syndrome coronavirus 2 (SARS Cov 2) which first appeared in Wuhan, China in December 2019. Genetic sequencing of this virus indicates that the virus is of Betacoronavirus type. which is closely related to the SARS virus. ${ }^{1}$ Although the vast majority of people who catch COVID-19 have only mild or uncomplicated disease, about 14\% suffer from serious illnesses requiring hospital care and oxygen support, 5\% need to be admitted to an intensive care unit. ${ }^{2}$ As many as half of the children with confirmed COVID-19 are treated in hospital and only $8 \%$ need intensive care and $4 \%$ die. Most of the cases of children are suspected asymptomatic, many are examined only because they have close contact with infected people. Infants younger than one month are more likely to need intensive care. The child who died was known to have a pre-existing congenital disease. The possibility of coinfection with other bacteria or viruses can increase the severity of symptoms in the child. ${ }^{2}$

The potential for excessive use of antibiotics in the COVID-19 pandemic era is a global threat to the increasing incidence of multiresistant bacteria. Many patients receive antimicrobial therapy even though in reality it has not been proven to be coinfected with bacteria or fungi. The rationale for the use of antibiotics in COVID-19 seems to refer to the experience of bacterial superinfection in influenza infection, which is generally caused by infection with Streptococcus 
pneumoniae and Staphylococcus aureus bacteria. Viral infection in the respiratory tract can be a predisposing factor for bacterial or fungal co-infection which can increase the severity of the disease until death. The incidence of bacterial co-infection in COVID-19 is still unknown. Recommendations for antibiotic administration vary between countries with the tendency to give empiric antibiotics due to the difficulty in ascertaining the cause of respiratory infection and detecting coinfection. On the other hand, the use of appropriate antibiotics in coinfection with bacteria provides a major benefit in killing germs. The macrolide class of antibiotics can act as prophylaxis for pneumococcal and staphylococcal bacterial infections. ${ }^{3}$

Certain antibiotics such as azithromycin in addition to killing bacteria also show anti-viral effectiveness, for example against the Zika virus, by reducing viability and proliferation of the virus. A randomized controlled trial (RCT) by Tahan F et al (2007) in children with bronchiolitis caused by respiratory syncytial virus (RSV) given clarithromycin was associated with reduced length of stay in hospital, oxygen requirements, treatment with $\beta 2$-agonists within six months. ${ }^{3}$

The purpose of this EBCR discussion is to determine the co-infection that occurs in children with COVID-19 and the effectiveness of antibiotics for co-infection in COVID-19.

\section{Case Report}

A boy aged 10 months 22 days came to the ENT-KL clinic for a tracheostomy cannula change plan. The patient only coughs occasionally if the secretions are heavy. The discharge from the tracheostomy appears thick white. No fever and shortness of breath. The patient had had tracheostomy since the age of 2 months when he was admitted to Neonate Intensive Care (NICU) with a diagnosis of Bronchopulmonary dysplasia (BPD) and grade 2 laryngomalasia. 5-6 times per day and nebulize $0.9 \% \mathrm{NaCl} 2-3$ times per day at home. The patient was then given a rapid test for the surgical procedure and the results were IgG SARS Cov2 reactive. Patients are referred to the child's PIE team for further evaluation. There is no history of contact with COVID-19 patients.

Physical examination showed the child was compos mentis, pulse: $118 \mathrm{x} /$ minute (sufficient content and tension), BP $70 / 50 \mathrm{mmHg}$, RR $34 \mathrm{x} /$ minute, $\mathrm{T} 36.7^{\circ} \mathrm{C}$, $\mathrm{SpO} 299 \%$ with water room. Body weight $11 \mathrm{~kg}$, length body $68 \mathrm{~cm}$. There was no chest retraction, vesicular breath sounds with coarse grants from both lung fields.

Laboratory tests showed HB 12.6 gr / dl RBC 4.67 x $10^{6} / \mathrm{mm}^{3}$ WBC $13.170 / \mathrm{mm}^{3} \mathrm{Ht} 37 \%$ PLT 355.000 / $\mathrm{mm}^{3}$ LED $17 \mathrm{~mm} \mathrm{/} \mathrm{hour} \mathrm{DC} \mathrm{0/5/22/63/10} \mathrm{ALC}$ 825.3 NLR 34.9 CRP $<5$. Chest X-ray showed right and left perihiller infiltrates. The results of rapid test of patients with non-reactive IgM and reactive IgG. The patient had 2 swab polymerase chain reaction (PCR) in a positive way.

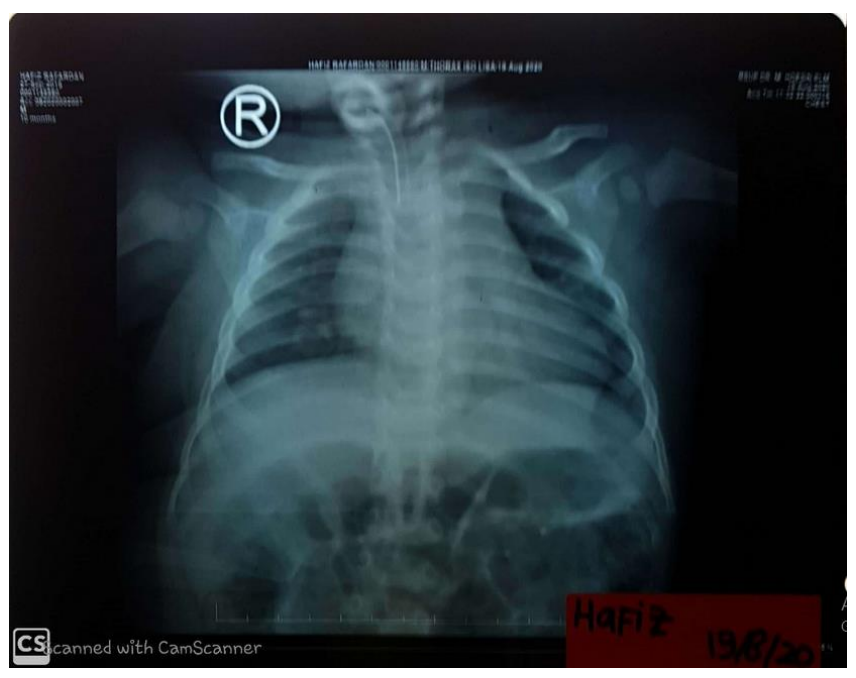

Figure 1. Chest X-ray of the patient.

\section{Problem}

Patients with COVID-19 confirmed coinfection with pneumonia and supported by clinical signs requiring appropriate antibiotic therapy. The incidence of coinfection accompanying children with COVID-19 cases is suspected to be high, ranging from mild to severe symptoms. Severe cases require antibiotics to prevent mortality and morbidity. In accordance with the clinical guidelines for the management of COVID-19 in children (June 2020 edition), ${ }^{4}$ antibiotics, both the macrolide group and the cefalosporin group, are given in coinfected COVID-19 cases. For this reason, this 
evidence-based case presentation aims to determine the effectiveness of giving antibiotics in cases of COVID19 and co-infection, especially pneumonia.

$\mathbf{P}$ : a COVID-19 patient has confirmed co-infection with pneumonia.

I : antibiotics

C : without antibiotics

$\mathbf{O}$ : therapeutic success

\section{Methods}

The literature search to answer the clinical problems mentioned above is to browse the literature online using the electronic database PubMed, Cochrane Library and Paediatrica Indonesiana in November 2020. The keywords used for the search were "COVID-19" "SARS Coronavirus 2". "children" . "Pediatric", "childhood") "respiratory coinfection", "coinfection", "antibiotics" or "azithromycin". , randomized clinical trials, meta-analyzes, systematic reviews, prospective and retrospective cohort trials and case reports. Based on the search method with the above criteria, 234 articles were obtained from PubMed, 688 Google scholar articles, 0 articles from Cochranese and 0 articles from Paediatrica Indonesiana. Then, the selection of relevant articles was carried out using the steps as shown in chart 1.

After further review, eight articles that were deemed relevant to the problem were selected, which then underwent a critical analysis to determine whether the articles were valid, important and applicable to patients. Furthermore, an assessment of levels of evidence was carried out based on the classification issued by the Oxford Center for Evidence-Based Medicine. There were three meta-analyzes, two systematic reviews, one randomized controlled trial, one single case, and one clinical trial. These publications are not randomized controlled trials examining the effect of antibiotics on coinfection of COVID-19 in children. In fact, there are no studies that directly link the effects of antibiotics in co-infected COVID-19 patients. For this reason, we divide separate discussions into two, namely: 1) regarding the characteristics of co-infection in COVID-19 patients and; 2) regarding the effect of antibiotics on the clinical symptoms of COVID-19 patients.

\section{Characteristics of co-infection in COVID-19}

There were four publications in the form of three meta-analyzes and one case series with quite a large number of subjects. ${ }^{5-8}$ The three metaanalyzes contained different studies. The case series research used was not included in the three meta-analyzes.

\section{Meta-analysis}

The search results obtained three metaanalysis studies regarding co-infection. The first report was published by Zheng et.al (April 2020), 7 conducted between January and April 2020. The aim of this study was to systematically review the condition and public health burden on children infected with SARS Cov 2. Inclusion criteria were pediatric patients confirmed by SARS Cov 2, research retrospective observational, case reports, or research articles describing the epidemiology, demographics and clinical features of confirmed cases with more than three patients. A total of 14 studies were included in this meta-analysis with a total of 410 children. Thirteen studies were conducted in China, two of the 13 studies were conducted in the same hospital and one study in Spain. Four studies analyzed co-infection with COVID-19 with a total of 272 children. The incidence of co-infection was found to be $10.14 \%$ (3.97-16.3\%). The most common cause of coinfection was Mycoplasma pneumoniae, which was 50\% (28.24\% -71.76\%) and Influenza / Parainfluenza virus, which was $22.76 \%(4.76 \%$ 40.77\%). Other co-infections include adenovirus, syncytial virus and Epstein-Barr virus. There was no significant heterogeneity in this study. The incidence of co-infection was not analyzed for 
clinical symptoms or outcome. 7

The second study was conducted by Lansbury et al. (May 2020), ${ }^{6}$ conducted between January 1 and April 172020 in pediatric and adult patients. The aim of this study was to evaluate the burden of coinfected patients with COVID-19. The included studies came from China, America, Spain, Thailand and Singapore. There were 3834 patients from 29 observational studies and one RCT. Divided into 27 studies reporting inpatient data (six of which are in ICU care) and two studies reporting mortality data for COVID-19 patients and one study reporting outpatient data. All studies in this meta-analysis are different from the meta studies. -analysis by Zheng et al (April 2020). Most of the sample population was adults and three studies included a sample of children (86 patients). Almost all patients (> 90\%) received empiric antibiotics, but they were not administered to the pediatric group due to mild symptoms. A total of 22 studies obtained coinfection data based on laboratory examinations, whereas in eight studies there was no clear information. Patients were grouped into ICU and combined ICU-inpatient. The overall rate of all studies included aimed at bacterial coinfection was $7 \%$ (95\% CI 3-12, $\mathrm{n}=2183,18$ studies, I2 $92.9 \%$ ). Subgroup analysis of the study with ICU data separation alone and combined was $14 \%$ (95\% CI 5-26, $\mathrm{n}=204$, I2 74.7\%) versus 4\% $(95 \%$ CI $1-9, \mathrm{n}=1979$, I2 91.7\% ). There were $3 \%$ of COVID-19 patients with laboratory confirmed coinfection of the virus (95\% CI 1-6, $\mathrm{n}=1014.16$ studies, 1262.3\%). Subgroup analysis showed no significant difference in the proportions between patients in the ICU and their combined 5\% $195 \%$ CI $1-14, \mathrm{n}=42.2$ studies) and $3 \%$ (95\% CI $1-5 \%$, $\mathrm{n}=972.14$ studies, I2 $62.9 \%$ ). Specific co-infected pathogens were identified in 17 studies (including studies of children). The most frequently detected bacterial pathogens were Mycoplasma pneumoniae (42\%), Pseudomonasaeruginosa $(12 \%)$ and Haemophilus influenzae (12\%). Other bacteria detected were Klebsiella pneumoniae (four patients), Enterobacter (three patients), Acinetobacter baumannii, Chlamydia, Enterococcus faecium, methicillin resistant Staphylococcus aureus (MRSA) and Serraria marscecens. Four fungal pathogens were identified in the adult study (none in the pediatric study), namely, Candida albicans, Aspergillus fumigatus and Candida glabrata. Respiratory viral co-infection was found in 14 studies including pediatric studies, such as RSV (16.9\%) followed by influenza A. S.aureus, $S$. pneumoniae or $S$. pyogenes which are also less. There is no evidence of an increased risk of death in co-infected COVID19 patients. These findings support the exclusion of empiric antibiotics in the majority of patients infected with SARS Cov2. 6

Hoang A et al (May 2020) 5 reported a systematic review with the aim of evaluating the characteristics of clinical, laboratory and radiological symptoms as well as drug therapy for children with COVID-19. The types of research included are cross sectional, case series, case control, case reports, review articles, opinion papers and letters to journal editors. Patients were enrolled up to 21 years of age with confirmed COVID-19. There were 131 relevant studies out of 1142 studies. The study came from 26 countries with the largest amount of data obtained from America, namely 2572 children. China contributed $64.1 \%$ of the study. The number of patients in this study was 7780 patients. There were 35 studies totaling 1183 children that specifically examined coinfection. There was coinfection in 72 children (5.6\%). Forty-five children were co-infected with bacteria, with the highest percentage being Mycoplasma pneumoniae (58.3\%), Enterobacter sepsis (2.8\%) and Streptococcus pneumoniae (1.4\%). Meanwhile, virus coinfection was found in 27 children including Influenza virus A / B (11.1\%), RSV (9.7\%), Cytomegalovirus (CMV) (4.2\%), Epstein-Barr virus (EBV) (4.2\%). Adenovirus (2.8\%), Human metapneumovirus (2.8\%) and Human parainfluenza virus (2.8\%). 5 


\section{Case Series}

One study by Zhu et al (April 2020), 8 with a large sample size of 257 patients. This research has not been included in the three meta-analysis studies above. Patient ages ranged from 2 to 99 years. It was found that $94.2 \%$ of patients had viral, bacterial and fungal coinfection. There were 81 patients $(31.5 \%)$ who had viral co-infection, 236 patients $(91.8 \%)$ had bacterial co-infection and 60 patients $(23.3 \%)$ had fungal co-infection. Bacterial pathogens include $S$. pneumoniae (59.5\%), Klebsiella pneumoniae (KP) (55.6\%), Haemophyllus influenza (40.1\%), Aspergillus (23.3\%), EBV (20.2\%), E. coli $(9.3 \%)$, S. aureus (8.2\%), HRV (4.7\%), P. aeruginosa (4.7\%), MC (4.3\%), HAdV (3, 9\%), HSV (3.1\%), A. baumannii (2.8\%), CP (2.5\%), Mucor (2.5\%), influenza B (1.9\%), MP (1.6\%), Bordetella pertussis (1.2\%), Candida (0.8\%), CMV (1.2\%), influenza A $(0.8 \%)$, $\mathrm{HBoV}(0.4 \%)$, Human pneumonia virus (HMPV) $(0.4 \%)$ and Cryptococcus (0.4\%). An analysis of the proportions of each co-infection was carried out based on the severity of COVID-19 symptoms. Patients with mild symptoms had bacterial coinfection (91.2\%), viral coinfection (31.5\%) followed by bacteria-virus (33.3\%), virus-fungi $(14.1 \%)$ and virus-bacteria-fungi (14.1\%). Patients with severe or critical symptoms had bacterial (94.1\%), viral (35.3\%), fungal (29.5\%) and bacterial-fungal $(29.5 \%)$ co-infection. There was no difference in the proportion of microorganisms based on the degree of disease severity. Coinfection varied with age and time of onset. Under the age of 15 years, 11 pathogens were found and HMPV was present only in this group. The coinfection rates for KP, Aspergillus, E. coli, and HRV were the lowest in this age group. Between the ages of 15 and 44 years, 22 influenza $A$ and $\mathrm{HBoV}$ pathogens were found only in this group. Baumannii, Mucor, influenza B and MP coinfections were the highest. 8

\section{Antibiotics in co-infection with COVID-19}

There are three studies that report on giving antibiotics to COVID-19 patients, namely two RCTs and one case series. Studies on antibiotic administration in COVID-19 assess clinical improvement regardless of co-infection status. There are also no specific studies on children.

\section{Clinical trial}

Furtado et al's (October 2020) ${ }^{9}$ RCT study, 9 conducted on adult patients with the aim of assessing the addition of azithromycin to standard care, including HCL, would improve clinical outcomes for patients hospitalized with severe COVID-19. There were 397 patients, divided into the azithromycin group with 214 patients and the control group with 183 patients. The study was conducted in Brazil. Inclusion criteria were a minimum age of 18 years with suspected or confirmed COVID-19 less than 14 days after onset. Patients included were classified as severe with at least one weight criteria, namely the use of oxygen supplements of more than $4 \mathrm{~L} / \mathrm{min}$; use of nasal cannula; use of non-invasive positive-pressure ventilation; or mechanical ventilation. Patients were divided into the HCL group and the HCL group with azithromycin. In conclusion, there was no significant difference between the azithromycin and HCL groups and the control group (HCL only) in terms of clinical improvement based on a sixpoint scoring system with OR 1.36 (95\% CI 0.941.97), $\mathrm{p}=0.11$ and in terms of mortality OR 1.08 (95\% CI 0.79-1.47), $\mathrm{p}=0.63$. These findings do not support the use of rutinazithromycin in combination with HCL. 9

The next study was an open label non randomized clinical trial conducted by Gautret et al. (March 2020), 10 with the inclusion criteria of patients over 12 years of age with confirmed PCR swabs. The subjects were divided into two groups, namely the control group and the intervention group. The control group with hydroxychloroquine (HCL) and the intervention group with a combination of azithromycin and 
hydroxychloroquine. Not explained in detail about the criteria for azithromycin administration, only mentioned based on "clinical decisions". In the control group, there were 16 subjects, and 20 patients in the intervention group. Pediatric patients were included in the control group because they had milder clinical symptoms. The results show that HCL therapy can reduce viral load in COVID-19 patients and this effect is strengthened by adding azithromycin. PCR results for the day of the HCL and azithromycin groups were negative on day 6 and significantly faster than the other groups $(p=0.002) \cdot 10$

\section{Case Series}

Million et al (April 2020), 11 conducted a study from 3 March 2020 to 31 March 2020 involving 1061 patients with COVID-19 in a French hospital who were treated with azithromycin and HCL for at least 3 days. The mean age of the patients was $43.6 \pm 15.6$ years. There were 350 patients excluded including six children $<14$ years of age. This study aims to assess the effectiveness of azithromycin and hydroxychloroquine. Only 10 $(0.9 \%)$ patients became critical and $8(0.75 \%)$ patients died. ${ }^{11}$

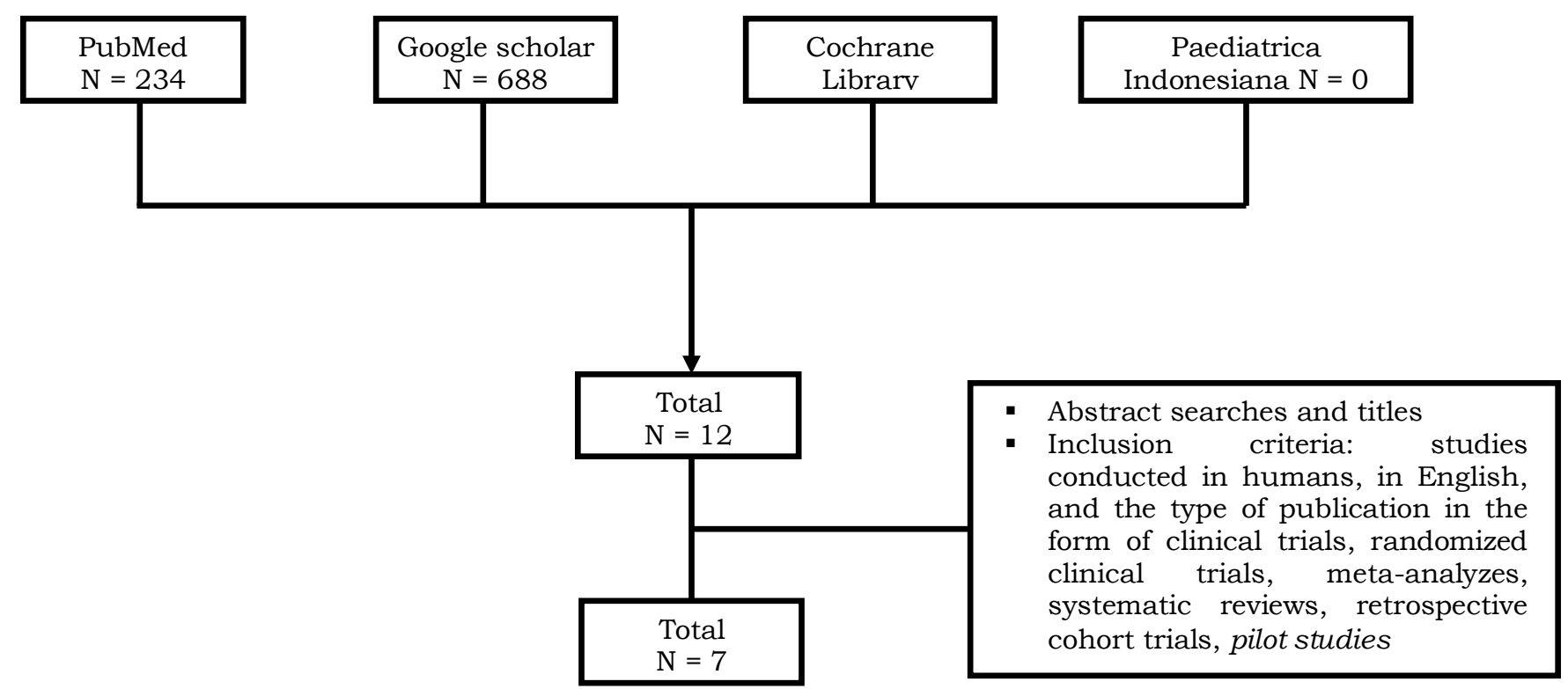

Chart 1. The flow of selecting articles that are considered relevant

Table 1. Critical case series review by Zhu et al.8

\begin{tabular}{ll}
\hline \multicolumn{1}{c}{ Criteria } & \multicolumn{1}{c}{ Explanation } \\
\hline Inclusion criteria are clear & All patients were positive RT-PCR swabs \\
$\begin{array}{l}\text { The diagnosis uses realible and valid standard } \\
\text { parameters }\end{array}$ & Diagnose COVID-19 by RT-PCR pharyngeal swab. \\
Taking consecutive cases & Yes \\
$\begin{array}{l}\text { Demographic reporting, clinical information, follow-up, } \\
\text { clear outcomes }\end{array}$ & $\begin{array}{l}\text { Patient demographics are reported. Follow up and } \\
\text { outcome were not clearly reported. }\end{array}$ \\
Use of appropriate statistical analysis & Chi Square test is used to find the number of \\
& coinfection in various age groups. \\
LOE & IIIb \\
\hline
\end{tabular}


Table 2. Critical analysis of research by Furtado (2020) and Gautret (2020). 9,10

\begin{tabular}{|c|c|c|}
\hline & Furtado $(2020)^{9}$ & Gautret (2020) ${ }^{10}$ \\
\hline Recruitment of subjects & $\begin{array}{l}\text { The characteristics of the two groups are } \\
\text { the same }\end{array}$ & $\begin{array}{l}\text { The characteristics of the two groups may } \\
\text { be different because the therapy group is } \\
\text { the chosen clinical decision. }\end{array}$ \\
\hline Allocation & $\begin{array}{l}\text { Block randomization was stratified by } \\
\text { study site, patient age and type of oxygen } \\
\text { therapy. }\end{array}$ & No randomization \\
\hline Maintenance & $\begin{array}{l}\text { All subjects received the same treatment. } \\
\text { A total of } 7 / 214 \text { in the therapy group and } \\
9 / 183 \text { in the control group dropped out. }\end{array}$ & $\begin{array}{l}\text { All subjects received the same treatment. } \\
6 \text { patients dropped out. }\end{array}$ \\
\hline Measurement results & $\begin{array}{l}\text { Clinical criteria include the need for } \\
\text { oxygen therapy and death. }\end{array}$ & PCR negative on day 6 . \\
\hline Result & $\begin{array}{l}\text { There were no significant differences } \\
\text { between the two groups. The } 95 \% \text { CI of } \\
\text { the RR is narrow. }\end{array}$ & $\begin{array}{l}\text { The proportion of negative PCR on day } 6 \\
\text { was higher in the HCL + azithromycin } \\
\text { group than in the HCL alone and non- } \\
\text { therapy groups }(100 \% \text { vs } 57.1 \% \text { vs } \\
12.5 \%)\end{array}$ \\
\hline LOE & $\mathrm{Ib}$ & Iib \\
\hline
\end{tabular}

Table 3.Critical review of Million et al's research (April 2020) 11

\begin{tabular}{ll}
\hline \multicolumn{1}{c}{ Criteria } & \multicolumn{1}{c}{ Explanation } \\
\hline $\begin{array}{l}\text { Inclusion criteria are clear } \\
\text { The diagnosis uses realible and valid standard } \\
\text { parameters }\end{array}$ & $\begin{array}{l}\text { All patients were positive RT-PCR swabs } \\
\text { Daking consecutive cases }\end{array}$ \\
$\begin{array}{l}\text { Demographic reporting, clinical information, follow-up, } \\
\text { clear outcomes }\end{array}$ & $\begin{array}{l}\text { Patient demographics are reported. Follow-up and } \\
\text { outcomes include improvement, intensive care and } \\
\text { death. } \\
\text { Tse of appropriate statistical analysis }\end{array}$ \\
TOE & $\begin{array}{l}\text { There was no special statistical test, only a descriptive } \\
\text { outcome proportion. }\end{array}$ \\
\hline
\end{tabular}

\section{Discussion}

The administration of antibiotics in COVID-19 in children has yet to be studied. Antibiotics are given to children with suspicion of coinfection. Antibiotics may be effective in children with pneumonia. Blood or sputum cultures can help diagnose pneumonia caused by bacteria. The use of broad-spectrum antibiotics is not recommended in mild cases, but may be considered in severe cases. Antiviral drugs have not been proven effective to date 12

Pneumonia is a acute respiratory infection that most often causes death in children in developing countries. Generally the cause of pneumonia is bacteria, especially Streptococcus pneumoniae, Haemophilusinfluenzae and Staphylococcus aureus which are responsive to beta-lactam. 13 There are also types of pneumonia that are not responsive to beta-lactam antibiotics which are classified as atypical pneumonia. Some of the known respiratory atypical bacteria are Mycoplasmapneumoniae, Chlamydia pneumoniae, Legionellapneumophila and Ureaplasma urealyticum. The empiric antibiotics given for atypical pneumonia include the macrolides, quinolones, and tetracyclines. ${ }^{14}$ 
The choice of macrolide antibiotics is an appropriate choice for pneumonia coinfection. Although there are several other options such as cefalosporin. Azithromycin is a macrolide group that can be given in a single dose with a duration of 5 days. Ferweda et al. (2020) in an RCT study found that the effectiveness of 3-day azithromycin administration was as effective as amoxciclav for 10 days in children with lower respiratory tract infections. Azithromycin side effects are also few and generally tolerable. 15

There are no studies that directly assess the effectiveness of antibiotics against coinfection in both children and adults with COVID-19. The existing studies only link the administration of antibiotics regardless of co-infection status.

The most common co-infection, based on reports that mostly came from China, was Mycoplasma pneumoniae followed by influenza or parainfluenza viruses. Based on the three metaanalyzes the coinfection rate ranged from $5.6 \%$ $14 \%$. 5-7 The incidence of coinfection appears to be higher in patients admitted to the ICU. Almost all studies included in this meta-analysis were retrospective studies. Although the number of subjects in the meta-analysis was quite large, the incidence of co-infection, especially in reports with pediatric subjects, was low, limiting the certainty of the evidence. None of the reports described the association of co-infection with clinical symptoms or outcome. All reports are limited to the incidence of co-infection and types of germs. The most common bacteria found were bacterial pathogens with the type Mycoplasma penumoniae $142 \%$ $58.2 \%)$, followed by influenza viruses $(11.1 \%$ $27.7 \%)$ and RSV (9.7\% - 16.9\%).

One case series by Zhu et al. Showed a very high co-infection rate of $94.2 \% .8$ It is likely that this difference was due to the different ranges of microorganisms examined. It also did not explain the clinical symptoms associated with coinfection in all studies. It is possible that the types of coinfection may differ according to geographic location depending on which microorganisms are prevalent in causing coinfection. For example, in Zhu's research, the most germs obtained were Streptococcus pneumoniae.

Antibiotics have shown an improvement in viral load, especially azithromycin. Azithromycin is known to have effectiveness against Mycoplasma pneumoniae. Azithromycin also has antiinflammatory and immunomodulating effects, therefore macrolides are chosen for the treatment of viral respiratory tract infections, including COVID-19. The immunomodulating activity of azithromycin acts in two phases, namely acute and chronic. In the acute phase, azithromsin reduces cytokine production, whereas in the chronic phase macrolides increase neutophil apoptosis and inhibits the production of IL- 1 beta, IL-6, IL-8 and TNF alpha in rhinovirus infections. Its abilities were also found to be effective against the Zika virus. ${ }^{3}$

Evidence regarding the benefits of giving antibiotics to COVID-19, especially in children, is still lacking. There was only one RCT that did not show a benefit of antibiotics. 9 One non-randomized therapeutic study did show a faster reduction in viral load, but the number of subjects was very limited.10 In addition, in all studies antibiotic administration was in combination with HCL. However, one case series study with a large number of subjects showed safety and good outcomes in the combination of azithromycin and HCL. ${ }^{11}$

\section{Conclusion}

This critical study shows that there is still very limited evidence regarding the administration of antibiotics in co-infection with COVID-19 in children, so we cannot conclude with certainty the benefits of giving azithromycin or other antibiotics. Research that only describes the findings of the causes of co-infection in COVID-19 which is quite high, but it is suspected that there is an effect of variations in different geographical locations. Local research is needed to determine the characteristics of co-infection according to the 
area of residence. However, given the high incidence of coinfection, the difficulty of testing to prove or rule out co-infection, antibiotics can be considered. Macrolides, especially azithromycin, are the antibiotics most frequently studied in COVID-19 and have activity against Mycoplasma pneumoniae and also have immunomudulatory effects.

\section{Suggestion}

The patient in this case report is a boy aged 10 months 22 days who was diagnosed with COVID19 based on PCR. Given the condition of patients with complications in the form of attached tracheostomy and grade II laryngomalasia who often receive treatment in connection with cannula, the patient can be considered for antibiotic administration. Although the evidence analysis did not find good data showing the clinical effectiveness of antibiotics, their administration could accelerate viral load reduction with good safety and outcome. Azithromycin, which is the most studied antibiotic in COVID-19 cases, can be considered for administration in this patient.

\section{References}

1. The Novel Coronavirus Pneumonia Emergency Response Epidemiology Team. The epidemiological characteristics of an outbreak of 2019 Novel Coronavirus Diseases (COVID19) - China, 2020. China CDC Weekly. 2020;2(8):113-22.

2. Götzinger F, Santiago-García B, NogueraJulián A, dkk. COVID-19 in children and adolescents in Europe: a multinational, multicentre cohort study. Lancet Child Adolesc Health. 2020;4(9):653-61.

3. Tahan F, Ozcan A, Koc N. Clarithromycin in the treatment of RSV bronchiolitis: a double-blind, randomised, placebo-controlled trial. Eur Respir J. 2006;29(1):91-7.

4. Tim Satuan Tugas COVID-19 PP IDAI.
Panduan Klinis Tatalaksana COVID-19 pada Anak. 14 Juni 2020. BP IDAI; 2020.

5. Hoang A, Chorath K, Moreira A, et al. COVID19 in 7780 pediatric patients: A systematic review. EClinicalMedicine. 2020;24:100433. DOI:https://doi.org/10.1016/j.eclinm.2020.1 00433.

6. Lansbury L, Lim B, Baskaran V, Lim WS. Coinfections in people with COVID-19: a systematic review and meta-analysis. J Infect. 2020;81(2):266-75.

7. Zheng B, Wang H, Yu C. An increasing public health burden arising from children infected with SARS-CoV2: A systematic review and meta-analysis. Pediatric Pulmonol. 2020:DOI:10.1002/ppul.25008.

8. Zhu X, Ge Y, Wu T, dkk. Co-infection with respiratory pathogens among COVID-2019 cases. Virus Res. 2020;285:198005doi: 10.1016/j.virusres.2020.198005

9. Furtado RHM, Berwanger O, Fonseca HA, dkk. Azithromycin in addition to standard of care versus standard of care alone in the treatment of patients admitted to the hospital with severe COVID-19 in Brazil (COALITION II): a randomised clinical trial. Lancet. 2020;396(10256):959-67.

10. Gautret P, Lagier J-C, Parola P, dkk. Hydroxychloroquine and azithromycin as a treatment of COVID-19: results of an openlabel non-randomized clinical trial. Int $\mathrm{J}$ Antimicrob Agents. 2020;56(1):105949doi: 10.1016/j.ijantimicag.2020.105949.

11. Million M, Lagier J-C, Gautret P, dkk. Early treatment of COVID-19 patients with hydroxychloroquine and azithromycin: A retrospective analysis of 1061 cases in Marseille, France. Travel Med Infect Dis. 2020;35:101738doi: 10.1016/j.tmaid.2020.101738

12. Chen Z-M, Fu J-F, Shu Q, dkk. Diagnosis and treatment recommendations for pediatric 
respiratory infection caused by the 2019 novel coronavirus. World J Pediatr. 2020;16(3):24046.

13. World Health Organization, Department of Maternal N Child and Adolescent Health, World Health Organization. Revised WHO Classification and Treatment of Pneumonia in Children at Health Facilities: Evidence Summaries.; 2014. Diakses 1 November 2020 Tersedia pada: http:/ / apps.who.int/iris/bitstream/10665/1 37319/1/9789241507813_eng.pdf

14. Lee H, Yun KW, Lee HJ, Choi EH. Antimicrobial therapy of macrolide-resistant Mycoplasma pneumoniae pneumonia in children. Expert Rev Anti-infect Ther. 2018;16(1):23-34.

15. Ferwerda A. Efficacy, safety and tolerability of 3 day azithromycin versus 10 day co-amoxiclav in the treatment of children with acute lower respiratory tract infections. J Antimicrob Chemother. 2001;47(4):441-46. 